\title{
ÉCRITURE, INSTITUTION ET SOCIÉTÉ LE TRAVAIL LITTÉRAIRE DANS LA COMPAGNIE DE JÉSUS EN FRANCE (1620-1720)
}

\author{
Stéphane VAN Damme
}

\begin{abstract}
«L'intellectuel jésuite est d'abord un missionnaire. Il parle, il écrit, il enseigne, mais son œuvre n'est qu'une contribution au dialogue, inauguré par saint Paul, de l'Église avec les cultures. "

Jean-Claude Dhotel, Les Jésuites de France. Chemins actuels d'une tradition sans rivage, Paris, 1985.
\end{abstract}

RÉSUmé : Depuis quelques années, un renouveau historiographique s'est amorcé dans le champ des recherches sur l'ancienne Compagnie de Jésus autour de la production des savoirs. Dans cet article, on s'est attaché à saisir la place, les modalités et les enjeux d'une reconnaissance des pratiques littéraires jésuites en France entre 1620 et 1720 , en essayant de montrer les tentatives de constitution d'un apostolat littéraire. Deux approches sont ici proposées : l'une strictement institutionnelle qui essaie de mesurer l'importance du travail intellectuel dans l'espace des charges à l'intérieur de l'Ordre; une autre davantage centrée sur la communication sociale de l'Ordre à travers la discussion de plusieurs dossiers de la polémique antijésuite.

MoTs-CLÉs : scriptor, pratiques littéraires, travail intellectuel.

ABSTRACT: For the past few years, a renewal of historical studies has begun on the intellectual work of the old Company of Jesus. In this paper, we would like to emphasize the place and the process of literary activities in France between 1620 and 1720, by describing the attempts to create an intellectual apostolate. Two approaches have been applied here : on the one hand, from an institutional point of view, we will estimate the importance of intellectual work in the spectrum of responsibilities inside the Congregation; on the other hand, the analysis based on several antijesuit polemics will point to the social communication of the Order.

KEYWORDS : scriptor, literary practices, intellectual work. 
ZUSAMMENFASSUNG : Seit einigen Jahren beschäftigt sich die historische Forschung mit der intellektuellen Aktivität der Gesellschaft Jesu. In diesem Artikel geht es um die Stellung und die Legitimation der literarischen Tätigkeit der Jesuiten in Frankreich zwischen 1620 und 1720, wobei gezeigt wird, wie man versucht hat, ein literarisches Apostolat zu begründen. Dabei wird ein doppelter Ansatz zugrunde gelegt : in einem institutionellen Ansatz wird die Bedeutung der intellektuellen Tätigkeit im Spektrum der verschiedenen Aufgaben innerhalb des Ordens behandelt, und in einem Ansatz, der sich auf die soziale Kommunikation konzentriert, werden verschiedene antijesuitische polemische Schriften untersucht.

STICHWÖRTER : Scriptor, literarische Praxis, geistige Arbeit.

RESUMEN : Desde hace algunos anos, ha empezado una renovación historiográfica en el campo de las investigaciones sobre la primera Compañia de Jesús y la producción de los saberes. Tratamos aqui de precisar el lugar, los modalidades y las implicaciones del reconocimiento de cierta forma de práctica literaria jesuita en Francia en los años 1620-1720 y de definir el intento de constituir un apostolado literario. Se proponen dos orientaciones: la primera, especificamente institucional, intenta medir la importancia del empeño intelectual dentro de los cargos o funciones internas a la Orden jesuita; la secunda está más bien centrada sobre la comunicación social de esta Orden, por medio del estudio de algunos documentos relativos a la polémica antijesuita del tiempo.

Palabras Claves : prácticas literarias, empeño intelectual, scriptor.

Stéphane VAN Damme, né en 1969, agrégé d'histoire, achève une thèse à l'université de Paris I sur le statut de l'auteur dans la Compagnie de Jésus en France et les milieux intellectuels provinciaux aux xvII et $\mathrm{xvil}^{e}$ siècles. Il est actuellement membre du Groupe de recherches interdisciplinaires d'histoire du littéraire à l'École des hautes études en sciences sociales et ATER au Collège de France.

Adresse : Collège de France, 11 place Marcelin-Berthelot, 75231 Paris Cedex 05.

Courrier électronique : stephane.vandamme@wanadoo.fr 
Dans une perspective qui cherche à réfléchir sur les rapports entre pratiques littéraires et institution religieuse ou plus spécifiquement, dans le cadre jésuite, sur la rencontre entre culture du collège et culture urbaine, la question de la publication est à retenir, car elle s'avère une des voies d'investigation fructueuse pour visiter à nouveaux frais les modalités d'intégration de la Compagnie de Jésus dans les sociétés urbaines de la France d'Ancien Régime, mais aussi pour comprendre comment un champ d'activité s'autonomise.

Dans ce vaste terrain d'études déjà profondément parcouru, on a choisi d'emprunter un itinéraire particulier : celui des auteurs jésuites. Cet angle d'attaque semble en effet avoir été négligé par une historiographie plus soucieuse de mettre en valeur les grandes figures d'écrivains de la Compagnie de Jésus que de s'intéresser aux spécificités de l'activité littéraire au sein de cette communauté. Trois lectures neuves du problème ont néanmoins encouragé l'ouverture d'un tel chantier historiographique. Les travaux de François de Dainville sur les corps d'écrivains ${ }^{1}$, de Michel de Certeau sur les mystiques ${ }^{2}$ ou de Marc Fumaroli sur les rhétoriciens ${ }^{3}$ ont ainsi mis l'accent sur les aspects institutionnels du travail littéraire dans l'Ordre, en essayant d'articuler la diversité des pratiques d'écriture qui surgissent au $\mathrm{XVII}^{\mathrm{e}}$ siècle avec les postures sociales de l'auteur jésuite et les mutations de l'Ordre. Prolongeant certaines de ces approches, les analyses pionnières de la production imprimée parisienne de Henri-Jean Martin ${ }^{4}$ ont permis de souligner l'existence de véritables politiques du livre jésuite dans le sillage de la Contre-Réforme et d'esquisser un espace éditorial de production et de diffusion, propre à l'institution, avec ses aires de circulation privilégiées et sa police du livre. Enfin, la sociologie du premier champ littéraire proposée par Alain Viala ${ }^{5}$, en reconnaissant la prépondérance des auteurs jésuites dans le milieu des gens de lettres au midi du siècle, a pu suggérer une

1. François de DaInvIlle, « Projet d'un corps d'écrivains à Toulouse en 1712 », Archivum Historicum Societatis Iesu, t. VII, 1938, p. 285-291.

2. Michel de Certeau, La Fable mystique. T. I : XVI -XVII siècle, Paris, Gallimard, 1982. Il faut rappeler que l'expression « travail littéraire » est de M. DE CERTEAU dans son article «Le dix-septième siècle français », in Les Jésuites. Spiritualité et activités. Jalons d'une histoire, Paris/Rome, Beauschesne/Centrum Ignatianum, 1974, p. 71-109.

3. Marc Fumarol, L'Âge de l'éloquence. Rhétorique et " res litteraria » de la Renaissance au seuil de l'époque classique, $1^{\text {re }}$ éd. 1980, Paris, Albin Michel, 1994, p. 247-256.

4. Henri-Jean MarTin, Livres, pouvoirs et société à Paris au xví siècle (1598-1701), Genève, Droz, 1969.

5. Alain Viala, La Naissance des institutions littéraires au xví siècle, Lille, Atelier national de reproduction des thèses, 1983, et ID., Naissance de l'écrivain. Sociologie de la littérature à l'âge classique, Paris, Minuit, 1985. 
approche en termes de trajectoire sociale, et situer les carrières littéraires jésuites dans l'espace social de la littérature.

Dans le cadre de cette étude, on souhaiterait précisément porter notre attention sur la question de l'émergence et de la reconnaissance de l'activité littéraire à la fois dans l'Ordre, et dans les sociétés urbaines. Complexe, cette activité s'avère tour à tour instrument et indice de fabrication du consensus que tente de réaliser la Compagnie de Jésus autour de son action multiforme durant trois siècles.

Parmi les nombreuses pistes de recherche en cours d'exploitation, on a choisi de suivre ici deux itinéraires d'enquête. Une approche strictement institutionnelle cherchera à comprendre comment s'est opérée la légitimation du travail littéraire et son intégration dans la grille des charges de la Compagnie. Une seconde voie privilégiera l'aspect conflictuel de la communication sociale de l'Ordre, en soulignant les négociations, les tensions, et les polémiques qui se trament autour de cet apostolat, peu à peu suspecté et rejeté des horizons familiers des activités de la Compagnie de Jésus ${ }^{6}$.

À ce stade de la recherche, il faut s'empresser de préciser les limites de notre terrain d'investigation. Géographiquement, loin de couvrir toute la Compagnie, et même tout le royaume de France, on procédera à l'examen de quelques sites et à des sondages : Lyon, Paris, Toulouse; chronologiquement : on laissera de côté la période de fondation pour s'intéresser au second siècle.

UN CORPS POUR L'ÉCRIT? VERS L'AUTONOMISATION DU TRAVAIL LITTÉRAIRE DANS LA COMPAGNIE DE JÉSUS

Au cours du Xvir ${ }^{e}$ siècle, une progressive différenciation des tâches au sein de la congrégation jésuite voit le jour ${ }^{7}$. Parmi ces nouvelles fonctions, le renforcement de l'apostolat missionnaire de l'intérieur tient une part prépondérante ${ }^{8}$. Et comme dans d'autres ordres religieux (bénédictins, etc.) à

6. Cette étude repose sur une pluralité d'approches documentaires. Elle est en partie centrée sur les grandes séries d'archives produites par la Compagnie et conservées à Rome et à Vanves. Il s'agit aussi bien des catalogues du personnel, des lettres annuelles que de la correspondance entretenue par les supérieurs des provinces de France avec les pères généraux. Ces sources seront présentées au fur et à mesure de leur exploitation.

7. À titre d'exemple, on renvoie ici aux analyses d'Adrien DemoustiER sur la province de Lyon, "Les catalogues du personnel de la province de Lyon en 1587, 1606 et 1636 ", Archivum Historicum Societatis Iesu, t. XLII, 1973 p. 3-105 et t. XLIII, 1974, p. 3-84 et, plus généralement, ID., "La distinction des fonctions et l'exercice du pouvoir selon les règles de la Compagnie de Jésus », in Les Jésuites à la Renaissance. Système éducatif et production du savoir, éd. Luce Giard, Paris, Presses universitaires de France, 1995, p. 3-34.

8. Voir, dans ce numéro, les articles consacrés à la mission, p. 335-380. 
la même époque, et sous l'impulsion de différents facteurs (nécessités de la controverse, de la théologie positive, etc.), le travail intellectuel tend à se constituer en apostolat autonome. Cette évolution est particulièrement nette chez les mauristes qui vont, autour de l'érudition historique, conforter la place de l'histoire dans l'épanouissement monastique du religieux ${ }^{9}$. Forts du «devoir d'intelligence ${ }^{10} »$, les jésuites ont-ils emprunté les mêmes voies, ou se sont-ils contentés d'instrumentaliser leurs pratiques d'écriture pour les mettre au service de tous les apostolats de l'Ordre? Cette question doit nous conduire à réévaluer la spécificité et l'identité du travail intellectuel dans la Compagnie de Jésus, souvent considérée comme originale et centrale dans le mouvement de la Réforme catholique.

Pour revisiter ce champ de recherche complexe, un chemin de traverse a pu sembler opportun. On s'intéressera ici non pas à tous les auteurs ayant appartenu à la Compagnie, en prenant pour critère la publication imprimée, mais à ceux que l'institution désigne de manière officielle par le titre de scriptor, d'écrivain. Il ne s'agit donc pas pour l'heure d'entreprendre une sociologie de l'activité littéraire, mais de scruter comment dans la division du travail qui affecte l'organisation interne de l'Ordre, un espace des pratiques d'écriture s'institutionnalise, et dans quelle mesure un champ apostolique s'autonomise.

\section{Estimation et répartition dans l'espace français}

La désignation de la qualité de scriptor dans l'espace des charges et des emplois à l'intérieur de la communauté jésuite en France ne résulte pas d'un processus linéaire et homogène sur l'ensemble du territoire de l'assistance de France ${ }^{11}$. Ne disposant pas d'un dénombrement général de ces effectifs, il a fallu procéder par sondage. À quatre dates : 1620, 1660, 1690, 1720 , l'examen des catalogues annuels du personnel des provinces de France, de Champagne, de Lyon, d'Aquitaine et de Toulouse, a souligné la faiblesse des échantillons concernés, puisque 5 jésuites ont cette qualification autour de 1620,4 en 1660,2 en 1690 et 7 en $1720^{12}$. Il en ressort

9. Voir Daniel-Odon Hurel, "Les bénédictins de Saint-Maur et l'histoire au xvII siècle ", Littératures classiques, «L'histoire au xvil siècle », 30, printemps 1997, p. 33-50, en part., p. 37-38.

10. Cette expression est empruntée à $L$. GIARD, «Le devoir d'intelligence ou l'insertion des jésuites dans le monde du savoir », in op. cit. supra n. 7, p. XI-LXXIx.

11. A. Demoustier, "La distinction des fonctions et l'exercice du pouvoir selon les règles de la Compagnie de Jésus », art. cit. supra $n$. 7.

12. Pour réaliser ces sondages, on s'est appuyé sur les fac-similés des catalogues annuels du personnel conservés à Vanves aux Archives des jésuites de France ( $=\mathrm{AJF}$ ), fonds Champagne, pour la province de Champagne, A 1, A 10, A 38, A 68; pour la province d'Aquitaine, A 302, A 305, A 308, A 311; pour la province de France, A 537, A 540, A 554, A 564; pour la province de Lyon, A 804, A 809, A 818, A 812; pour la province de Toulouse, A 1055, A 1058, A 1061, A 1063. 
une impression de forte concentration de ces écrivains dans un type de résidence : le collège, au détriment d'autres structures. Ainsi, ils ne sont jamais accueillis dans des résidences de mission, dans les maisons professes ou les noviciats. De plus, l'implantation des écrivains se fait préférentiellement dans les "grands" collèges de la Compagnie de Jésus en France (à Bordeaux, Lyon, Paris, Toulouse). À première vue, la géographie des scriptores n'est pas le fait du hasard, elle met en évidence leur présence sur les fronts de catholicité de la France méridionale (en 1660, on trouve un scriptor à Montpellier, en 1619, au collège de Saintonge). Enfin, le tropisme parisien s'accentue au $\mathrm{XVIII}^{\mathrm{e}}$ siècle, puisque l'essentiel des écrivains résident au collège de Paris.

Afin de compléter ces coupes transversales par une étude longitudinale, trois sites ont été retenus plus particulièrement, qui permettent d'articuler trois échelles d'analyse différentes : le collège de Clermont à Paris, le collège de la Trinité de Lyon et la province jésuite de Lyon ${ }^{13}$.

$\mathrm{Au} \mathrm{Xv}^{\mathrm{e}}$ siècle, on ne trouve pas trace dans les catalogues parisiens de l'appellation d'écrivain, les premières mentions datent de 1606 dans le catalogue du personnel du collège de Clermont, c'est-à-dire trois ans après le rétablissement de la Compagnie en France, par l'édit de Rouen de 1603. Dans les deux premiers tiers du XVII ${ }^{e}$ siècle, le nombre des scriptores reste modeste (en moyenne : 2 à 3 postes par an), avec parfois des lacunes : entre 1652 et 1658, pas de postes. Après 1633, on note une recrudescence, puis sous le règne de Louis XIV, une forte croissance : on passe de 5 postes entre 1667 et 1679 à 8 entre 1680 et 1752 , avec plusieurs pics, 12 à 15 entre 1712 et 1718 , et 10 à 14 entre 1742 et 1747 .

Le collège de la Trinité à Lyon ne se situe pas au même niveau. Entre 1635 et 1725 , on a une moyenne de 1 poste par an, avec parfois des lacunes, comme par exemple entre 1635 et 1665 . À partir de 1670 , les effectifs demeurent très fluctuants même si le nombre de postes croît sensiblement pour atteindre le seuil de 4 par an. Puis entre 1680 et 1700 , on constate une nouvelle baisse, suivie d'une reprise entre 1700 et 1725 , avec un pic de 4 postes en 1710 .

À l'échelle d'une province tout entière, en l'occurrence celle de Lyon qui rassemble tous les établissements jésuites du Sud-Est du royaume de France, de la Franche-Comté et de la Savoie, l'examen des catalogues livre

13. Pour Paris, l'essentiel de l'information est tiré de l'étude de Gustave Dupont-FerRIER, La Vie quotidienne d'un collège parisien pendant plus de 350 ans. Du collège de Clermont au lycée Louis-le-Grand, Paris, De Boccard, 1921, t. I, p. 59-62, et t. III, p. 31-38 et 126; pour Lyon, on s'appuie sur l'examen des catalogues annuels et triennaux du personnel de la province de Lyon, Archives romaines de la Compagnie de Jésus (= ARSI), lugd. 14-16 pour la période 1600-1725, et Lugd. 19-25. Ces catalogues ont été partiellement reproduits et sont conservés aux AJF, fonds Champagne, mss 806-819, « Cat. Breves prov. lugd. », 1636-1725; et mss $A 855,858,860,870$ pour les catalogues triennaux. 
des résultats qui confortent le portrait d'ensemble. À trois dates : en 1633 , sur 3 scriptores, 2 sont affectés à la Trinité, 1 à Vienne; en 1665, 5 sur 6 nommés résident à Lyon, et 1 à Embrun; en 1705, sur un total de 4, tous sont employés à Lyon.

Bien que très sommaires, ces chiffres soulignent une forte polarisation de l'activité littéraire autour des grands établissements de la Compagnie, due à une concentration des ressources financières, des équipements culturels (bibliothèques, observatoires, etc.) et du personnel.

\section{Trajectoires institutionnelles des scriptores}

Pour Paris et Lyon, on dispose d'indications pour cerner le profil de ces écrivains et reconstituer l'horizon des choix qui a présidé à leur nomination.

Premier constat d'importance : l'âge d'accès à cette fonction reste fort inégal, et donc peu soumis à l'inscription dans un cursus des fonctions ou dans une carrière intellectuelle. Au collège de Clermont, sur 70 cas étudiés aux $\mathrm{XVII}^{\mathrm{e}}$ et $\mathrm{XVIII}^{\mathrm{e}}$ siècles, les âges se répartissent de la façon suivante : 13 jésuites (soit $19 \%$ ) ont entre 31 et 38 ans au moment de leur entrée en poste, 28 (soit 40,5\%), entre 40 et 49 ans; 12 (soit $17 \%$ ) entre 50 et 59 ans; 12 (soit $17 \%$ ) entre 60 et 69 ans. Après 70 ans, les exemples se font rares, mais ne sont pas négligeables, puisqu'ils sont encore 5 jésuites à dépasser cet âge ( 2 avaient 73 ans, 1 autre 79 ans, 2 plus de 80 ans). Par conséquent, l'office de scriptor s'affirme d'après ces chiffres comme un emploi d'âge mûr, même s'il n'est pas à Paris, contrairement à Lyon, une retraite pour la vieillesse. Le P. Claude-François Ménestrier ne deviendra «écrivain» dans la cité rhodanienne qu'à l'âge de 69 ans après avoir publié plus de cent trente titres.

La durée d'exercice de la charge n'offre pas plus de stabilité. À Paris, 13 scriptores restèrent seulement 1 an (soit 18,5\%); 24 (soit 34,3\%) occupèrent ce poste pour une durée comprise entre 2 et 5 ans; 16 jésuites (soit 22,9\%) entre 6 et 10 ans; 22 (soit 31,5\%) plus de 10 ans. Toutefois, certains s'y employèrent pendant presque toute leur vie dans la Compagnie. Ainsi le P. Claude Buffier, entre 1701 et $\mathbf{1 7 3 5}$, ou le P. Thomas Du Pré entre 1715 et 1757 , y restèrent pendant 35 ans. De même, les PP. Fronton Du Duc ou Dominique Bouhours gardèrent leur fonction sans changement pendant 18 et 32 années respectivement. De ces deux premiers éléments d'appréciation, on peut conclure à une très grande diversité des postures d'écrivains à l'intérieur de l'Ordre.

Dès lors, si ces critères ne semblent pas dessiner un profil homogène, il est bon de se demander comment s'exerçait cette charge. Représentait-elle, dans l'espace institutionnel, une activité à part entière, ou était-elle subor- 
donnée à d'autres apostolats? À cet égard, il est remarquable de noter les situations opposées de Paris et de Lyon. Au collège de la Trinité, le statut d'écrivain n'est que très rarement une charge que l'on exerce exclusivement, les scriptores ont souvent la responsabilité de la bibliothèque, ou cumulent une fonction de direction spirituelle (confession ou congrégation mariale, comme par exemple les PP. Théophile Raynaud, ou Jean Croiset qui dirigeront la congrégation des Messieurs de Lyon). Plus rares sont ceux qui exercent un enseignement. À Paris, le travail littéraire a précocement conquis son indépendance : quatre jésuites seulement cumulent cette fonction avec un autre office ${ }^{14}$.

Comme le laissait supposer l'étude de l'âge d'accès et de la durée d'exercice, la fonction de scriptor ne constitue souvent qu'un moment dans la carrière. À Paris, elle intervient majoritairement après une expérience longue d'enseignement. La presque totalité a en effet enseigné avant, mais avec des profils très différents : trois jésuites se sont consacrés aux mathématiques, six aux Saintes Écritures et dix n'ont enseigné que la rhétorique. Le primat conféré à la rhétorique renvoie à une production d'éloges et de traités d'éloquence qui vont assurer dans la première moitié du xvII siècle, la réputation du collège parisien. À Lyon, la trajectoire du P. Jacques Georges, scriptor à Vienne à 63 ans, né à Bourges, illustre bien cet aspect transitoire de la fonction. Maître ès arts, il a enseigné successivement la rhétorique pendant cinq ans, la philosophie pendant trois ans, les mathématiques pendant quatre ans, la théologie positive pendant deux ans, et a ensuite entamé une longue carrière de prédicateur pendant trente-cinq ans ${ }^{15}$. De même, parmi les écrivains dont la carrière n'est pas achevée, nombreux seront ceux qui seront appelés après ce poste à occuper dans le collège des fonctions importantes. Si une majorité d'anciens scriptores revient dans le circuit de l'enseignement ${ }^{16}$, ce sont les charges administratives les plus hautes dans la hiérarchie des pouvoirs qui s'offrent aux plus capables ${ }^{17}$.

Au total, le passage par l'emploi d'écrivain est donc loin de constituer une impasse dans la carrière du jésuite. En même temps, l'extrême diversité des âges et des durées d'exercice ne traduit pas l'inscription dans une carrière intellectuelle commune. Plusieurs traits caractérisent ainsi leur

14. Denis Petau est professeur d'Écriture sainte (1625-1627), Gabriel Cossart, bibliothécaire (1659-1672), Claude Buffier, professeur d'histoire et géographie (1701-1737), et Guillaume-François Berthier, professeur d'Écriture sainte.

15. Sur Jacques Georges, voir ARSI, Lugd. 19, catalogue triennal « second» de la province de Lyon, 1633, $\mathrm{n}^{\circ} 3, \mathrm{f}^{\circ} 72$, et Carlos Sommervogel, Bibliothèque de la Compagnie de Jésus, Bruxelles/Paris, Oscar Schepens/Alphonse Picard, 1890-1930, t. III, col. 1338-1339.

16. À Paris, 5 sont affectés à une chaire de théologie; 1 à une chaire de thétorique; 2 à la bibliothèque.

17. Deux écrivains deviendront recteurs; un provincial. 
situation à Paris. Le nombre de postes est extrêmement fluctuant d'une année sur l'autre, ce qui confère à cette spécialisation un caractère temporaire et transitoire. Ils constituent à côté d'autres charges plus stables dans la grille des emplois, une sorte de détachement provisoire. Les premières expressions (en particulier en 1619, les formules «scriptioni vacat» ou «vacat editioni ») utilisées dans les catalogues confortent cette impression. Le temps de l'écriture est encore conçu comme une vacation, une occupation secondaire ${ }^{18}$. De plus, fidèle à un principe de nomination répandu dans la Compagnie, c'est l'homme qui fait le poste et non l'inverse. S'il n'y a pas de jésuite engagé dans un travail d'écriture nécessitant un allègement de son emploi du temps, le poste est laissé vacant.

En vérifiant la corrélation entre le genre dominant pratiqué avant l'affectation et le recrutement, pourrait-on aller plus loin et esquisser les grandes lignes d'une politique des auteurs? Rien n'est moins sûr. En effet, comme le suggère l'exemple du $\mathrm{P}$. Georges, l'adéquation entre parcours d'écrivain et motifs d'affectation, n'est pas toujours évidente. Auteur de trois discours d'éloges funèbres publiés en 1610,1615 et 1622 , il ne devient écrivain qu'en fin de carrière, lorsqu'il travaillait à la rédaction d'un manuel de grammaire sur les «Problèmes en langue française ». De même, les catalogues triennaux "seconds" du personnel, qui reproduisent les appréciations du supérieur et donnent une justification des aptitudes pour les affectations futures, enregistrent rarement des précisions suffisantes, et de manière souvent aléatoire. Ainsi, parmi les quatre écrivains inscrits à Lyon en 1636, seul le P. Pierre Labbé, né en 1595, est chargé explicitement d'écrire des éloges (ad elogia scribenda). Pour la grande majorité, il faut se contenter de mettre en parallèle la production manuscrite et imprimée avec la trajectoire interne du jésuite si l'on veut approcher au plus près les catégories de jugement des supérieurs et les procédures de sélection des écrivains. À Lyon, les scriptores sont attachés à la confection d'ouvrages religieux, soit de controverse ou de spiritualité pratique comme le P. Joseph de Gibalin, soit d'hagiographie ou de théologie positive comme le P. Raynaud, soit d'histoire profane ou religieuse pour les PP. Jean Columbi ou Claude-François Ménestrier. Dans le cas du P. Dominique de Colonia, l'examen croisé des catalogues et de sa bibliographie ${ }^{19}$ révèle que son affectation à un poste de scriptor en 1705 correspond à un changement radical d'orientation dans sa production littéraire. Auteur dramaturge et professeur de rhétorique pendant la première partie de sa carrière, il se convertit à l'enseignement de la théologie positive et à l'écriture

18. Daniel Roche, «L'intellectuel au travail », in Les Républicains des Lettres. Gens de culture et Lumières, Paris, Fayard, 1988.

19. Sur la première mention de Dominique de Colonia comme écrivain, ARSI, Lugd. 23, catalogue triennal « second » de la province de Lyon, $1705, \mathrm{n}^{\circ} 15, \mathrm{f}^{\mathrm{os}} 395 \mathrm{v}^{\mathrm{o}}, 443 \mathrm{v}^{\circ}$. 
d'ouvrages de controverse après sa nomination à un poste d'écrivain. Bien qu'il soit présomptueux de généraliser à partir de cet exemple, on voit bien, malgré des réserves de méthode, que des données prosopographiques peuvent mettre à jour des dynamiques aveugles, et aider à mieux comprendre une instrumentalisation possible des auteurs dans la Compagnie de Jésus, ce qui jusque-là n'était suggéré qu'à partir de l'étude de la seule production.

LES OPÉRATIONS DE TRADUCTION DANS LE LANGAGE DU CORPS

Accompagnant la définition d'un nouveau statut religieux et social à l'intérieur de l'institution, on voit peu à peu se former « une stratégie d'énonciation qui cherche à dresser la carte d'une identité ${ }^{20} »$. Dans le cadre de l'analyse de l'apostolat missionnaire, des travaux récents ont insisté sur les entreprises de justification interne et externe de l'activité qui tendaient à masquer a posteriori les hésitations et les éventuels échecs de l'Ordre $^{21}$. De ce fait, ils ont contribué à reformuler l'interrogation sur les dispositifs de légitimation de l'action de la Compagnie, et sur les formes identitaires qu'elle revendique.

Une reconnaissance élémentaire:

l'incorporation aux pratiques administratives

Ainsi, la reconnaissance du travail littéraire ne se détermine pas à travers des déclarations de principe, mais par un lent processus d'incorporation qui s'opère tacitement dans les pratiques rédactionnelles administratives ${ }^{22}$. Le choix de cette source comme indice et trace d'une institutionnalisation implique que soit retenue l'idée que la Compagnie de Jésus, au-delà d'une

20. Luc Boltanski, Les Cadres. La formation d'un groupe social, Paris, Minuit, 1982, p. 30 .

21. Sur ce point, voir Bernard DompNIER, « La Compagnie de Jésus et la mission de l'intérieur ", in Les Jésuites et la civilisation du baroque, 1540-1640, sous la dir. de Luce GIARD et de Louis DE VAuCELles, Grenoble, Jérôme Millon, 1996, p. 155-179, mais aussi Charlotte DE CASTELNEAU, «Les ouvriers d'une vigne stérile. » Les jésuites et la conversion des Indiens au Brésil, 1580-1620, thèse de doctorat, Paris, École des hautes études en sciences sociales, 1999.

22. Un travail similaire de distinction s'est opéré pour les missions de l'intérieur par rapport aux tâches de prédication comme l'a analysé $\mathbf{B}$. Dompnier pour la province de Lyon : voir B. DOMPNIER, "L'activité missionnaire des jésuites de la province de Lyon dans la première moitié du xvır siècle. Essai d'analyse des Catalogi ", Mélanges de l'École française de Rome. Moyen Âge et Temps modernes, t. XCVII, vol. II, 1985, p. 941-959. 
inscription dans des pratiques sociales, est aussi une "communauté textuelle ${ }^{23}$ ").

Concernant la province de Lyon, à deux reprises, la documentation administrative enregistre ce changement. D'abord, dans l'un des quatre catalogues du personnel de la province envoyés à Rome, le troisième catalogue triennal qui donne des informations sur la gestion du temporel des différents collèges, on trouve en 1649 , deux feuillets qui ont été rajoutés et qui comportent la liste des ouvrages imprimés dans la province entre 1643 et 1649 , soit soixante-dix titres ${ }^{24}$.

Ensuite, c'est la conservation dans un autre type de document, les lettres annuelles, véritables comptes rendus des activités de la Compagnie dans chaque province pour le $P$. Général, d'une liste de la production littéraire du collège de la Trinité ${ }^{25}$. Ici, ce qui frappe c'est la situation de coprésence de cette description avec celle des autres apostolats (éducatifs, prédications, missionnaires...).

Ces deux indicateurs isolés qui n'ont pas donné suite à une pratique régulière et courante interrogent doublement cette reconnaissance de l'activité éditoriale. En premier lieu, les listes publiées ne se limitent pas à la production des seuls scriptores, les ouvrages des autres auteurs sont inventoriés, ce qui tend à montrer une relative institutionnalisation de pratiques littéraires informelles et invisibles dans l'Ordre. En second lieu, les livres recensés ne se cantonnent pas au registre religieux. Par exemple en 1696, on mentionne aussi bien les livres de mathématiques ou de navigation du P. Paul Hoste ${ }^{26}$, les ouvrages historiques ou le Traité sur les loteries du P. Claude-François Ménestrier que les livres de théologie du P. JeanFrançois Malatra ${ }^{27}$. La place que tiennent les ouvrages profanes, et en particulier la production scolaire, témoigne d'un glissement dans les préoccupations de la Compagnie.

Par ailleurs, il n'est pas anodin de relever que ces tentatives marginales d'intégration de l'activité dans les pratiques rédactionnelles administratives interviennent entre 1649 et 1700 , période durant laquelle la production imprimée se diversifie, où en particulier les livres à usage pédagogique

23. Nous empruntons cette notion à : Brian STock, The Implications of literacy. Written language and models of interpretation, Princeton, Princeton University Press, 1983. Il s'agit de montrer comment des pratiques d'écriture peuvent être à l'origine de la création d'une organisation sociale.

24. ARSI, Lugd. 19, catalogue triennal «tertius» de la province de Lyon, 1649, $\mathrm{f}^{\text {os }} 407 \mathrm{a}-407 \mathrm{~b}$.

25. ARSI, « Litterae annuae provincia Lugdunensus », 1696-1700, Lugd. 30, $\mathrm{f}^{\circ} 385 \mathrm{v}^{\circ}$.

26. Paul Hoste, L'Art des armées navales, ou traités des évolutions navales, Lyon, Anisson et Posuel, 1697.

27. Jean-François MaLATRA, Specimen Theologiae duodecim libris comprenhensae quibus ad calcem accedent aliqui tractatus ad universam theologiam pertinentes, Lyon, J. Thioly, 1698. 
envahissent le corpus. Enfin, si l'on observe les formes littéraires que prend cette codification écrite du travail littéraire, on constate une «littérarisation » de l'activité. En effet, alors qu'en 1649, on a affaire à une vision comptable, à un inventaire, la liste est préférée au récit, en revanche en 1696 , c'est la narration qui prime, soulignant de ce fait un alignement sur le modèle d'écriture employé pour décrire les autres activités ${ }^{28}$. Du catalogue du temporel aux lettres annuelles, en cinquante ans une première acclimatation semble avoir réussi.

\section{Rationalisation et instrumentalisation}

Une troisième approche doit venir compléter ce tableau d'ensemble. Ce sont les efforts de la Compagnie destinés à rationaliser l'utilisation de ces écrivains. Ils se déploient principalement dans deux directions.

Dès 1608, en effet, une première impression d'un Catalogus écrit par le P. Pedro de Ribadeneyra ${ }^{29}$ commence la tradition des bibliographies jésuites. Remanié à Lyon en 1609 par le P. Michel Coyssard, et à Anvers en 1613 par le P. André Schott, il va connaître une diffusion européenne. Cette œuvre sera poursuivie à Rome par deux continuateurs : le P. Philippe Alegambe qui, avec la collaboration du P. Jean Bollandus, publie en 1643, la Bibliotheca d'Anvers, et par le P. Nathanel Southwell ${ }^{30}$ qui fait paraître sa bibliographie des auteurs à Rome en 1676. Après cette date, des équipes se mettent en place à Rome avec le P. Filippo Buonanni, à Paris avec le $P$. René de Tournemine, et à Dijon avec le P. François Oudin, mais n'aboutiront pas à une nouvelle édition ${ }^{31}$. Pourtant, sur le désir de Rome, toutes les provinces de l'Ordre doivent désormais dresser des catalogues d'auteurs sous la forme de suppléments joints aux catalogues triennaux. Ainsi, le P. Oudin disposera de la liste complète des scriptores de 1675 à 1725 , son successeur à partir de 1725 , le $\mathrm{P}$. Jean-Louis Courtois recevra lui aussi de nombreuses listes ${ }^{32}$. Sur cette base, des bibliographies par province sont ébauchées : on a conservé ainsi le dictionnaire des auteurs du P. Henri Rybeyrete qui recense tous les auteurs vivants de la province de France

28. Sur la dimension cognitive de ce passage, voir Jack Goody, La Raison graphique. La domestication de la pensée sauvage, Paris, Minuit, 1979.

29. Pedro de Ribadeneyra, Catalogus scriptorum religionis Societatis lesu, secunda editio, plurimorum scriptorum accessione locupletior, Anvers, 1613.

30. Philippe Alegambe, Bibliotheca scriptorum Societatis Iesu, Anvers, Jean Meurs, 1643; Nathanel SoutrweLl, Bibliotheca scriptorum Societatis Iesu, Rome, J. de Lazzeri Varesi, 1676.

31. «Buonanni ", in C. Sommervogel, op. cit. supra n. 15, t. II, col. 383, G.

32. L'essentiel de l'information provient de M. Dykmans, "Introduction », in C. SommerVOGEL, op. cit. supra n. 15, t. XII, p. I-IV. 
entre 1640 et 1670 , et est adressé au provincial, le P. Étienne Deschamps. On compte dans l'index cent quatre-vingt-quinze noms ${ }^{33}$.

La diffusion de l'usage de la bibliographie dans la Compagnie de Jésus peut être mise en relation avec l'implantation des réseaux provinciaux de bibliothèques de collèges ${ }^{34}$, qui visent à rendre plus efficace la formation spirituelle et intellectuelle ${ }^{35}$. Elle est contemporaine de l'émergence de deux autres tendances manifestes de la production imprimée jésuite : la multiplication des bibliothèques «sans murs ${ }^{36}$ " (celles des PP. Antoine Possevin, Claude Clément ou Claude-François Ménestrier ${ }^{37}$ ) donnant des indications sur la manière de constituer une bibliothèque; et la diffusion de guides bibliographiques à destination des prédicateurs ou des membres des congrégations mariales (la Bibliotheca anti-janseniana du P. Labbé, la Bibliothèque des prédicateurs du P. Vincent Houdry, ou la Bibliothèque des jansénistes ${ }^{38}$ du P. de Colonia).

Il se manifeste aussi une volonté de mieux distribuer la présence des écrivains sur tout le territoire de l'Assistance. À ce sujet, le P. de Dainville a publié en 1938 un document rare qu'il a trouvé dans les archives

33. AJF, recueil Rybeyrete, 2 vol. manuscrits, le premier volume contient un dictionnaire des auteurs de la province de France, « Scriptores provinciae Franciae Societatis lesus ab anno 1640 ad annum 1670 », signalé par C. SommervoGeL, op. cit. supra n. 15, t. VIII, col. 341.

34. Paul MEch, «Les bibliothèques de la Compagnie de Jésus », in Histoire des bibliothèques de France. T. II : Les bibliothèques sous l'Ancien Régime, 1530-1789, sous la dir. de Claude Jolly, Paris, Promodis, 1988, p. 57-62; Dominique Julia, « La constitution des bibliothèques de collèges. Remarques de méthode ", Revue d'histoire de l'Église de France, t. LXXXIII, 1997, p. 145-161. C'est un champ d'enquête qui reste à encourager.

35. Sur cette question, Stéphane $V_{A N}$ DAMME, «Devenir enseignant de collège au XviI siècle. Itinéraires et expériences de formation des professeurs et régents jésuites de la province de Lyon ", Cahiers d'histoire. Revue d'histoire critique, 71, 1998, p. 37-54.

36. L'expression est empruntée à Roger CHARTrer, "Bibliothèques sans murs ", in ID., Culture écrite et société. L'ordre des livres (xIV-xvmi siècle), Paris, Albin Michel, 1996, p. 107-131. Pour un premier inventaire du genre, voir C. SoMmErvogel, op. cit. supra n. 15, t. X, col. 1902-1906. On ne compte pas moins de 10 titres pour le Xvir siècle, dont 6 d'auteurs français.

37. Antoine Possevin, Bibliotheca selecta qua agitur de ratione studiorum, in disciplinis, in salute omnium procuranda, Rome, 1693; Claude CLÉMENT, Musei, sive Bibliothecae tam privatae Extructio, Instructio, cura, Lyon, J. Prost, 1635; Claude-François Ménestrier, Bibliothèque curieuse et instructive de divers ouvrages anciens et modernes de littérature et des arts, ouverts pour les personnes qui aiment les lettres, Lyon, 1705. Sur les auteurs cités, on lira Robert DAMIEN, in Bibliothèque et État. Naissance d'une raison politique dans la France du xvif siècle, Paris, Presses universitaires de France, 1995, p. 85-89. Par ailleurs, d'autres études ont été consacrées au texte de Ménestrier : Noémi Hepp, "Entre le siècle de l'honnête homme et le siècle du philosophe. La Bibliothèque curieuse et instructive du $\mathrm{P}$. Ménestrier \#, Revue française d'histoire du livre, IIf s., 48, 1979, p. 737-746; et S. VAN DAMME, « Les livres du P. Claude-François Ménestrier (1631-1705) et leur cheminement ", Revue d'histoire moderne et contemporaine, vol. XLII, 1 , janv.-mars 1995, p. 5-45, en part., p. 31-32.

38. Philippe LABBE, Bibliotheca anti-janseniana, sive Catalogus piorum erudiorumque scriptorum, Paris, Cramoisy, 1645 ; Vincent Houdry, La Bibliothèque des prédicateurs, 8 vol., Paris, 1712; Dominique dE ColontA, La Bibliothèque des jansénistes, Lyon, 1722. 
romaines ${ }^{39}$. Il s'agit d'une lettre adressée au P. Général Michel-Ange Tamburini par le P. Jean-Joseph Guibert, visiteur de la province de Toulouse, le 7 mars 1712, qui concerne les conditions d'installation d'un groupe d'écrivains à Toulouse. Après avoir passé en revue les points concernant les ressources matérielles et les hommes, "les consulteurs assemblés par le P. Guibert ont examiné en quel lieu il convenait d'installer un corps d'écrivains ». L'attention portée au site géographique et institutionnel est intéressante, parce qu'elle souligne l'enracinement de l'apostolat intellectuel dans les collèges : " [...] d'un avis unanime, on jugea que le collège de Toulouse était un lieu très convenable et même le seul lieu que l'on pût désigner pour cette fondation ${ }^{40}$. $\gg$

$\mathrm{Ce}$ document témoigne d'une double évolution de la congrégation jésuite. D'abord, il met en scène un rapport centre/périphérie, qui n'est plus celui traditionnel Rome/province, mais qui joue désormais sur l'antagonisme français Paris/province ${ }^{41}$. En effet, ce constat rejoint d'autres analyses de la diffusion des modèles culturels à l'intérieur de l'Ordre dans l'espace français dès le début du xvII siècle, qui relèvent la nationalisation des recrutements et des productions intellectuelles. Celle-ci ne rend plus compte de la dynamique de la Compagnie de Jésus à l'échelle internationale caractérisée par une expansion géographique, et une volonté de renforcement de la centralisation de l'Ordre ${ }^{42}$. Ainsi, le développement du statut d'écrivain se conçoit dans le cadre d'une provincialisation du corps, censée réagir aux dangers occasionnés par le tropisme parisien des auteurs jésuites. Dès 1669, dans une lettre du Général au provincial de Toulouse, le R.P. Jean-Paul Oliva avait d'ailleurs dû interdire les déplacements sans permission à Paris, tant était grande l'attraction exercée par la capitale ${ }^{43}$. Par ailleurs, dans le contexte des créations académiques de la France méridionale, on peut se demander s'il ne cherchait pas à restaurer l'influence

39. F. DE Dainville, art. cit. supra n. 1, p. 285-291.

40. ARSI, Gal. $64, \mathrm{f}^{\mathrm{D}} 137$, lettre du 7 mars 1712 , cité par F. DE DAINUILLe, art. cit. supra n. 1 , p. 286.

41. Pour une mise en contexte de cette dynamique culturelle de l'espace français, on lira l'analyse essentielle de D. RocHE, "Le Royaume réglé. Paris et les provinces », in ID., La France des Lumières, Paris, Fayard, 1993, p. 190-223.

42. Il faut souligner ici les apports des travaux d'Antonella Romano, La Contre-Réforme mathématique. Constitution et diffusion d'une culture mathématique jésuite à la Renaissance (1560-1640), Rome, École française de Rome, 1999.

43. ARSI, lettres des généraux aux provinciaux de Toulouse, p. 178, cité par F. DE DAINviLe, art. cit. supra n. 1, p. 291. Par ailleurs, les suppléments des catalogues annuels qui recensent les jésuites placés hors de leur province d'origine confirment cette peur de la fuite des intellectuels toulousains : alors qu'en 1622-1623, ils ne sont que 2 sur 9 à venir de Toulouse, en 1709-1720, ils sont 7 sur 25. Voir AJF, fonds Champagne, catalogue annuel de la province de France, mss A 537, A 564. 
intellectuelle des jésuites sur les élites sociales. L'essor du jansénisme provincial peut donner raison à cette seconde hypothèse ${ }^{44}$.

En second lieu, à travers les différentes recommandations du P. Général, se dévoilent les procédures de sélection d'un groupe d'écrivains. La liste provisoire des jésuites toulousains susceptibles de devenir scriptores, qui est proposée, croise un certain nombre de critères qui nous éclairent sur les modalités du recrutement. Ainsi, le P. Michel Mourgues est retenu parce qu'il reçoit déjà de la ville de Toulouse une pension de six cents livres de gages annuels ce qui garantit l'autonomie du groupe vis-à-vis du Collège. Mais au-delà de l'aspect financier, les supérieurs accordent beaucoup d'intérêt à la réputation des auteurs. En la matière, la publication de livres qualifiés d'œuvres "non communes », c'est-à-dire d'ouvrages qui n'apparaissent pas comme des textes collectifs, constitue un facteur discriminant. Autrement dit, il faut à ce nouveau groupe une identité intellectuelle forte, de manière à susciter les éventuelles vocations littéraires chez les jeunes jésuites qui seront amenés à prendre la relève.

Ce projet ne vit jamais le jour, et seul un jésuite, le P. Jacques Vannière, reçut dans les catalogues suivants le titre de scriptor. Pour le P. de Dainville, les conditions financières n'auraient pu être remplies.

À travers cet échec de la généralisation de l'expérience parisienne, on saisit que le travail littéraire reste pour la majorité l'occupation d'auteurs non scriptores. La portée de ce constat n'est pas mince : l'essentiel de la construction de la figure sociale de l'homme de lettres jésuite s'est ainsi fait en dehors de toute visibilité institutionnelle. Ce sont donc les pratiques informelles, soumises à des influences multiples, qui ont permis cette maturation.

Pour autant, il ne faudrait pas négliger les conséquences de la création de la catégorie d'écrivain. Cette innovation autorise une meilleure instrumentalisation du travail littéraire qui jusque-là pouvait être laissé à l'initiative individuelle. Elle traduit peut-être, au sein de la gamme des statuts, une volonté de structurer des activités menées en privé, de leur donner une visibilité interne. En cela, elle constitue pour l'ensemble des auteurs un pôle de référence identitaire, en particulier dans la perspective d'une justification apostolique du service de plume. Ainsi, ce surgissement de la désignation de scriptor dans les archives de l'Ordre s'inscrit-il dans un vaste mouvement de travail de la Compagnie de Jésus sur elle-même. Afin de pouvoir surmonter l'expansion géographique et la multiplication des apostolats, la communauté se normalise, elle forge les instruments de sa représentation et de son dénombrement. Autrement dit, comme l'écrit M. de Certeau, « une

44. F. DE Dainville, art. cit. supra n. 1, p. 290-291. 
logique de l'intériorité fait alors contrepoids à celle de la dissémination apostolique ${ }^{45}{ }^{\prime}$.

CRISES, TENSIONS ET POLÉMIQUES AU DÉBUT DU XVIII ${ }^{\mathfrak{e}}$ SIÈCLE :

L'ACTIVITÉ LITTÉRAIRE JÉSUITE CONTESTÉE?

Malgré l'expansion rapide et la diversification du domaine littéraire jésuite au $\mathrm{xVII}^{\mathrm{e}}$ siècle $^{46}$, l'activité d'écriture et de publication sera constamment soumise à de violentes critiques venant aussi bien des rangs de la Compagnie de Jésus que de la société civile. En rouvrant le dossier de l'abondante littérature polémique antijésuite à l'époque moderne, il ne s'agit pas ici de mesurer la place qu'occupe la question de la publication dans un lexique de l'antijésuitisme en apparence stable, mais de repérer, à un moment clef de son histoire, la fin du règne de Louis XIV et la Régence, quelques-uns des motifs présents dans ces mauvais discours qui finissent par jouer en retour sur les efforts de banalisation d'un apostolat intellectuel et littéraire. On s'en tiendra ici à l'exposé de deux formes de dénonciation : l'une interne qui s'épanouit dans les sphères parisiennes de la Compagnie de Jésus, l'autre publique qui passe par le prisme de la production satirique.

\section{À Paris : la survie d'un corps d'écrivains en question}

La première polémique examinée ressort d'un conflit opposant dans la province de France en 1719, le P. Jacques Philippe Lallemant ${ }^{47}$, qui fait figure de représentant des écrivains parisiens et le P. Honoré Gaillard ${ }^{48}$, recteur du collège Louis-le-Grand. Cet affrontement par lettres interposées, arbitré par le R.P. Général, reformule les conditions d'émancipation du tra-

45. M. de CerteAu, « La réforme intérieure au temps d'Aquaviva ", in Les Jésuites, op. cit. supra n. 2, p. 64.

46. H.-J. MARTIN, op. cit. supra n. 4.

47. Jacques Philippe LaLlemant (1660-1748), auteur de vingt-six ouvrages, a entretenu une correspondance avec Fénelon, et a eu une activité intense de controversiste; il est l'auteur des Réflexions morales avec des Notes sur le Nouveau Testament traduit en françois, 4 vol., Paris, Le Comte et Montalant, 1713, voir C. Sommervogel, op. cit. supra n. 15, t. IV, col. 1387 1400.

48. Honoré Gaillard (1641-1727) fut précepteur du prince de Turenne, avant d'entamer une carrière de prédicateur à la cour entre 1702 et 1716. Il fut ensuite recteur du collège de Paris. Il a publié sept titres, pour l'essentiel des éloges funèbres, voir C. Sommervogel, op. cit. supra n. 15, t. III. 
vail littéraire au sein de la communauté jésuite et porte précisément sur deux aspects emblématiques de cette activité. D'abord, le P. Gaillard vise la circulation illicite de livres entre les maisons, qui échappe au contrôle des supérieurs (provincial, recteurs, préfets des études...) :

« Voicy ce que j'appris hier de manière à m'y faire faire plus de réflexion que je n'en avais fait. Cela consiste en deux faits. Premier fait. Je scavois bien qu'il $y$ avait une correspondance entre le $P$. Lallemant et le $P$. Longueval ${ }^{49}$ et que de la maison professe, il y avait souvent icy des messages du P. Lallemant au $P$. Longueval; mais ce que j'appris hier c'est qu'il y a pour cela un commissionnaire à gages, qui vient icy tous les jours au matin, porte des paquets et en rapporte ordinairement au $P$. Longueval et souvent au P. Dupré, et même vient-il quelquefois plus d'une fois par jour. C'est-à-dire que cette association s'entretiens [sic] fort librement, que tous les manèges continuent qu'il n'y a qu'elle qui ait ces commerces de lettres et de messages de chaque jour, et qui par la entretient ses complots.

Deuxième fait. Un colporteur étant venu se présenter à acheter à des pères de la maison professe quelques imprimés fautifs et de contrebande touchant la Constitution, on avait trouvé qu'il vouloit les vendre trop chers. [...] Voyez comment ce trafique [sic] d'argent se continue ${ }^{50}$."

La menace décrite par le P. Gaillard, qui renvoie à un type d'échanges d'information savante et de nouvelles commun à toute la « République des Lettres $^{51}$ ", interroge les normes de la lecture collective et de la possession du livre dans la Congrégation, et entre en conflit avec un idéal de la communication discrète et surveillée qui fonde les règles de l'épistolarité jésuite $^{52}$. En second lieu, il stigmatise la création d'un groupe autonome au sein de l'institution qui se soustrait peu à peu aux contraintes de la vie commune, comme le souligne l'expression « fort librement ». Le vocabulaire et les procédés polémiques employés brocardent le stéréotype de l'amitié préférentielle qui est un des topiques du discours moral des réformateurs des abus du clergé ${ }^{53}$.

49. Jacques Longueval (1680-1735) professa cinq ans les humanités à Amiens et, à La Flèche, quatre ans la théologie positive et l'Écriture sainte. Il prit part à la querelle autour du jansénisme, par son Traité du schisme (1718) qui lui valut une affectation en province. Il a publié cinq titres : voir C. Sommervogel, op. cit. supra n. 15, t. IV, col. 1935.

50. AJF, collection Prat, t. 78, lettre du P. Gaillard, recteur du collège de Paris, au P. Général, 22 mai 1719 , p. 173-174.

51. Voir Krzysztof Pomian, «De la lettre au périodique. La circulation des informations dans le milieu des historiens au xvil ${ }^{\mathrm{e}}$ siècle ", Organon, t. X, 1974, p. 25-43, et Paul Dibon, "Les échanges épistolaires dans l'Europe savante du xvir siècle ", Revue de synthèse, t. XCVII, 81-82, 1976, p. 31-50.

52. Sur la question des modèles de correspondance, voir L. GIARD, « Introduction (aux lettres et instructions) ", in IGNACE DE LoYOLA, Écrits, trad. du latin et éd. sous la dir. de Maurice Gruliani, Paris, Desclée De Brouwer, 1991, p. 619-627; et Dominique BerTrand, La Politique de saint Ignace de Loyola. L'analyse sociale, Paris, Cerf, 1985.

53. Sur ce point, voir le remarquable article de D. JuL1A, « Le prêtre ", in L'Homme des Lumières, sous la dir. de Michel Vovelle, Paris, Seuil, 1997, p. 391-429. 
Le système de défense du $\mathrm{P}$. Lallemant en date du 7 juin, consiste à désamorcer la menace, en proposant une triple justification de son action. D'abord, le complot qui reposerait sur la collusion d'un certain nombre de jésuites est désormais sans fondement en raison de la dispersion du groupe ou de son vieillissement.

«Ce n'est plus au reste la petite société qu'on poursuit : ce n'en est proprement que le phantôme [sic]. Elle est réduite aujourd'hui à si peu de chose qu'on ne comprend pas qu'elle excite encore la jalousie. [...] La mort nous a enlevé le P. Germon ${ }^{54}$, le P. Languedoc ${ }^{55}$ est bibliothécaire au collège, il est d'ailleurs mourant, et incapable de s'appliquer à des études sérieuses. En un mot, il y a trois ans que je ne l'occupe à quoyque ce soit. Le P. de Fontenay ${ }^{56}$ n'est plus des nôtres : le $P$. provincial en a disposé depuis neuf mois pour faire un employ aux pensionnaires. Le P. Du Tertre est averti depuis six mois pour enseigner la théologie, et il s'y prépare. Il ne reste donc plus au collège que le P. Longueval et le P. Du Pré ${ }^{57}$. Oserais-je demander à Votre Paternité ce qu'on reproche à ces Deux Pères pour les chasser ignominieusement de Paris, comme on veut le faire ${ }^{58}$ ?»

Le deuxième point de l'argumentaire porte sur le régime d'indépendance relative des auteurs jésuites ${ }^{59}$. Il est, selon le $P$. Lallemant, la conséquence d'une mise à l'écart des autres activités, induite par la recherche érudite. Or cette tendance n'est nullement préjudiciable à l'Ordre, puisque le produit fini, le livre, reste soumis à l'appareil de censure. Enfin, en déclinant les différents domaines d'intervention de l'Ordre (controverse antijanséniste, érudition biblique, rédaction d'ouvrages scolaires...) qui nécessitent une mise en imprimé, l'inventaire des travaux en cours de publication conforte l'existence de ce secteur apostolique. Une rhétorique utilitaire vient donc en contrepoint de la dénonciation d'un complot intérieur.

54. Barthélémi Germon (1663-1718), auteur d'un Traité théologique sur les 101 propositions énoncées dans la bulle Unigenitus, Paris, 1722.

55. Michel LANGUEDOC (1670-1742) professa la philosophie, la théologie morale et positive. Entre 1718 et 1728, il fut chargé de la bibliothèque du collège de Paris, il est par ailleurs l'auteur des notes insérées dans les cinq premiers volumes des Réflexions morales avec des Notes sur le Nouveau Testament traduit en françois, op. cit. supra n. 47.

56. Pierre Claude Fontenal (1683-1742) collabore aux Mémoires de Trévoux, et il a en patticulier signé le compte rendu de l'Histoire gallicane du P. Longueval, voir C. Sommervoged, op. cit. supra n. 15 , t. III, col. 855.

57. Thomas Du Pré (1680-1758) est l'auteur de trois titres dont un traité de théologie en 1722.

58. AJF, collection Prat, t. LXXVIII, lettre du P. Lallemant au P. Général, Paris, 7 juin 1719 , op. cit. supra n. 50 , p. 175-179.

59. Même si elle n'est pas abordée dans ce texte, la question firancière a régulièrement posé problème. Ainsi en 1695 , dans une lettre, le $P$. Général avait insisté auprès du provincial de France pour que les «écrivains de Paris » qui menaient le combat contre le jansénisme fussent pourvus du nécessaire, ARSI, Gal. 9, $\mathrm{f}^{\mathrm{0}} 243 \mathrm{v}^{\circ}$, lettre du 2 août 1695. 
«Selon vos ordres, mon V. R. Père, ils ne se croiront désormais dispensés de se mêler en rien de la Constitution. Ils ne feront plus ni livres, ni mémoires sur cette affaire. Mais qu'il leur soit permis de travailler à d'autres ouvrages selon leur vocation et leur talens. Le P. Du Pré, conjointement avec le P. Germon, avait faict [mot illisible] de l'ordre des Evêques acceptans un grand ouvrage dogmatique sur les 101 propositions ordinaires, où ils sont employés pour deux ans. Cet ouvrage qui est de deux gros volumes s'imprime actuellement à Paris avec la permission de M. Le Régent, et c'est le cardinal de Bissy qui le fait imprimer à ses frais.

[Par ailleurs, ils ont un projet de géographie universelle.] Il n'y a qu'à Paris où cet ouvrage puisse être continué, à cause de la quantité de livres qui est nécessaire pour cela et de près de 400 cartes qu'il faut faire graver sous les yeux. [...] Je n'ay encore donné au public que sept volumes des Réflexions et des notes sur le Nouveau Testament. Il nous en reste trois à faire imprimer. [...] Cet ouvrage est utile, et j'ose dire nécessaire à la religion. V. P. voudrait-elle prendre sur elle, de l'interrompre, et d'empescher qu'il fut achevé ${ }^{60}$ ? "

À travers cet exemple de polémique interne sur l'apostolat littéraire ou intellectuel, se lit une tension récurrente entre deux stratégies divergentes $^{61}$ : celle de l'expansion du champ apostolique qui passe ici par une diversification de la production littéraire jésuite, et celle d'un repli sur des activités légitimes, mais qui n'accordent aux écrivains qu'un statut d'auteurs occasionnels ${ }^{62}$ et à l'imprimé qu'un usage limité et instrumentalisé. Déjà en 1682, le P. Ménestrier se plaignait de ne pouvoir écrire ce qu'il voulait :

" Ainsi, Monseigneur, ne considérez cet ouvrage si éloigné de cette Profession, que comme ces ouvrages que quelques Pères de l'Église ont fait autrefois pour se divertir, quand ils ont traité de la Musique, de la Poésie, de l'Histoire, et des spectacles de leur temps. C'est ce qui pourra me justifier auprès de certains esprits, qui voudroient que l'on n'écrivit que des Livres Spirituels quand on est de profession à instruire le public des devoirs du Salut et de la Religion, mais comme il m'est indifférent de plaire à ces gens là, ou d'attirer leur censure, je seray satisfait de mon ouvrage, s'il peut vous marquer le respect avec lequel je suis, Monseigneur, votre très-Humble et très obéissant serviteur ${ }^{63}$. »

La fin du texte du P. Lallemant saisit bien les impasses et les présupposés de la politique menée par le P. Gaillard.

60. AJF, collection Prat, t. LXXVIII, lettre citée supra n. 58.

61. M. DE CERTEAU, art. cit. supra n. 45 , a très bien mis en évidence au début du XVII siècle sous le généralat de Claude Aquaviva, les termes de ce clivage.

62. On rejoint, dans ce cadre-là, la classification de A. VIALA, Naissance de l'écrivain, op. cit. supra n. 5, p. 179.

63. C.-F. Ménestrier, « ̀̀ Monseigneur le duc d'Aumont», in Des ballets anciens et modernes selon les règles du théâtre, Paris, Guignard, 1682, non paginé. 
« Je scay que l'idée du P. Gaillard est qu'il n'y ait point d'escrivains dans la Compagnie, mais seulement des prédicateurs, des confesseurs et des régens. Heureusement, ce Père n'est pas le maître chez nous pour y établir son ridicule système. Il l'appuie sur ce que les écrivains font des affaires à la Compagnie. Mais les prédicateurs ne luy en font-ils point? Elle donne des censeurs aux écrivains, c'est de quoi remédier au mal. Elle est obligée d'abandonner les prédicateurs à leur discrétion; et souvent elle ne s'en trouve pas mieux. Je pardonne du reste au P. Gaillard de faire cas du mestier de prédicateur, dont il a scu [sic] si bien profiter en tout sens, mais qu'il en fasse grâce aux pauvres écrivains, qui communément mènent une vie dure et peu agréable. Après tout, les sermons passent et les bons livres demeurent. L'histoire du P. Daniel et d'autres ouvrages seront lus lorsqu'on ne sçaura seulement s'il y a eu un P. Gaillard au monde ${ }^{64}$. »

En filigrane, se dessinent ici, les contours d'un apostolat qui se veut exemplaire. En insistant sur l'image du travail collectif, sur le rayonnement et la pérennité de l'imprimé, et sur le contrôle par une instance extérieure, la censure, le $\mathrm{P}$. Lallemant cherche à amplifier la portée négative des mesures prises par le Recteur.

Quelques années après le projet de 1712 , on est donc loin de la création d'un corps d'écrivains en province. Si on ne sait pas malheureusement ce que le $\mathrm{P}$. Général a répondu aux deux protagonistes, il n'est pas anodin de souligner les effets du contexte politique et religieux qui entoure cette affaire interne, et qui pèse sur ces tentatives de remodelage du champ apostolique jésuite. La fin du règne de Louis XIV comme la Régence ont, semble-t-il, vu se déstabiliser et se fragiliser le réseau des soutiens et des alliances qu'avait tissé la Compagnie de Jésus à la cour ${ }^{65}$. La lutte contre le jansénisme va cristalliser et radicaliser la critique antijésuite, en même temps qu'elle va faire de l'auteur jésuite un type littéraire ${ }^{66}$.

\section{La condamnation morale de l'écrivain jésuite}

Cet antagonisme semble d'autant plus difficile à surmonter qu'il est relayé, sur un autre registre, par une dénonciation publique. En effet, en réaction à l'attitude mondaine de certains auteurs jésuites tels les PP. Dominique Bouhours, René Rapin, Claude-François Ménestrier ou

64. AJF, collection Prat, t. LXXVIII, lettre citée supra n. 58.

65. Voir Georges Gurton, S.J., Le Père de La Chaize, confesseur de Louis XIV, Paris, Beauchesne, 1959.

66. Sur le contexte littéraire de cette polémique et les stratégies jansénistes, voir Catherine MAIRE, De la cause de Dieu à la cause de la nation. Le jansénisme au XVIIf siècle, Paris, Gallimard, 1998. 
Pierre Le Moyne, qui exercent leur ministère à la cour, toute une production satirique a vu le jour, donnant lieu à une circulation souvent manuscrite $^{67}$, parfois imprimée mais toujours clandestine, qui se propose de dévoiler les stratagèmes de l'Ordre et d'étaler les preuves de l'immoralité de ses écrivains. Sans revenir sur l'originalité ou la nouveauté de ces thèmes qui, pour la plupart, courent tout au long du XVII siècle dans la littérature pamphlétaire, il a pu sembler intéressant de scruter les représentations des pratiques d'écriture que ces textes mettent en œuvre.

Pour ce faire, l'attention s'est portée sur un ouvrage imprimé en 1719, conservé à la réserve des imprimés de la Bibliothèque nationale de France, au titre énigmatique, Jean danse mieux que Pierre, Pierre danse mieux que Jean, ils dansent bien tous les deux, qui met en scène le dialogue dans l'audelà de trois jésuites, les PP. François de La Chaize, Dominique Bouhours et Claude-François Ménestrier ${ }^{68}$.

Le premier intérêt de ce texte tient à l'expression de la dénonciation sous la forme littéraire mondaine du dialogue, et sur le mode fictionnel. Il autorise une circulation sur le marché des imprimés de large diffusion.

En second lieu, la condamnation morale qui donne tout son sens à cette conversation, s'appuie sur trois séries d'attaques. Tout d'abord, la production littéraire n'est pas gratuite, mais vise à s'assurer le soutien des Grands.

«Le P. Ménestrier :

“ C'est vous, qui avez prétendu le beau nom de Père et de source de Pensées Ingénieuses, par des Livres que vous en avez fait expressément à qui ce malheur est arrivé de ne pouvoir plus en enfanter, et faire valoir les talens de votre bel Esprit à la Gloire de vos Mécènes, et de ceux qui vous les paîoient si libéralement.

«Le P. Bouhours :

«Et qui sont ces mécènes, et ces Rémunérateurs si libéraux de mes louanges? Grâce à Dieu, à mon bon destin, et au crédit que mon savoir faire m'avoit acquis dans la Compagnie et dehors, je n'ai eu besoin que de moimême, pour mener une vie également douce et glorieuse dans le monde. [...]

67. Les fonds anciens des bibliothèques municipales ont conservé de nombreux recueils de poésie satirique; on peut citer, à titre d'exemple, celui de la Bibliothèque municipale de Lyon, ms. 757 (667), "Poésies contre les jésuites", qui comporte un épigramme sur les auteurs jésuites, "Aux RR PP jésuites-Autheurs du Journal de Trévoux », $\mathrm{f}^{\circ} 24 \mathrm{v}^{0}$.

68. Jean danse mieux que Pierre, Pierre danse mieux que Jean, ils dansent bien tous les deux, 5 vol., Tétonville, 1719 , in-12. L'usage de la fausse adresse "Tétonville ", ainsi que la vignette pornographique en début d'ouvrage avec la légende « Mon Père mettez votre instrument entre mes jambes » renvoient à une diffusion clandestine. L'éditeur indique dans une note : “ Ce texte a d'abord été imprimé à l'étranger à Cologne chez Pierre Marteau en 1714 ", fausse adresse connue. Ce livre a sans doute remporté un certain succès puisqu'il est édité à plusieurs reprises sous le titre Les Passetemps des jésuites ou les entretiens des PP. Bouhours et Ménestrier sur les défauts de leur Compagnie, 3 t., à Pampelune, chez les frères Ignace, 1721 (BNF, Ld 39 272). 
«Le P. Bouhours :

« J'ai vécu comme je devois vivre, et j'ai suivi sans m'écarter la route que tiennent les habiles gens de nostre Compagnie, de lui faire honneur en lui attachant les personnes de qualité par la voie de l'esprit, en le faisant paroître dans les livres, ou dans les Conversations ${ }^{69}$. »

Ensuite, les PP. Ménestrier et Bouhours constatent la perversion de cet apostolat (débauche, flatterie des auteurs).

«P. Ménestrier :

«Toutes sortes de livres ne font pas honneur à une personne ou à une Société Religieuse; et on vous a fait voir, qu'un livre, semblable à celui de vos Entretiens, est véritablement l'Ouvrage d'un débauché, et d'un frippon, qui exprime ses pensées toutes mondaines, sous le nom de galanterie, qualité ellemême très indigne à un Religieux, toute pardonnable qu'elle puisse être à un Courtisan et à un homme du monde. Allez, allez relire ce que Cléante vous dit là-dessus, et ce que le $\mathrm{P}$. Quesnel répondit à l'avertissement que vous mites à la tête de la troisième Edition de votre Lettre à un seigneur de la Cour, et convainquez-vous de l'idée qu'avoit tout le monde, hormis vous, de la vie effeminée que vous avez menée, et de la folle présomption que vous aviez d'être l'Arbitre de la Langue, et l'Echo des beaux Parleurs de la Cour et des Ruelles. Ne m'obligez pas à parler davantage et laissez vivre dans l'Hôpital des Orphelins le fruit de vos leçons, et de votre éloquence à persuader le sexe, que la galanterie n'est pas un si grand mal. [...]

«P. Ménestrier :

"Oseriez-vous dire, que par vos discours et par cette sorte de commerce, vous aïez produit le moindre changement favorable à la piété, non pas même un semblable à celui que nôtre P. Bourdaloue causa aux étoffes, dont les Dames se servoient pour habiller leur vanité [...].

«P. Bouhours :

«Quelle idée avez-vous donné de votre équité et de votre désintéressement par les louanges outrées, et le plus souvent fondées sur la flatterie toute pure, et sur l'évidence contraire, dont vous avez composé la vie du Roi par Médaille $^{70}$ ? »

Enfin, redoublant l'accusation d'hypocrisie, le dialogue soutient que l'usurpation de l'identité d'auteur est une pratique qui remonte à Ignace de Loyola.

«P. Ménestrier :

«Oui je dis qu'il y a des gens, qui soutiennent très sérieusement qu'il n'en fut jamais l'Auteur, et qu'il le reçut entier et parfait des mains de son confes-

69. Voir Les Passetemps des jésuites, op. cit. supra n. 68, p. 2-4.

70. Ibid., p. 5-8. 
seur Don Garcie de Cisneros, auquel s'étant adressé pour faire sa confession générale, quand, résolu de se donner à Dieu, il alla pour cet effet au Couvent de Montserrat en Catalogne ${ }^{71}$."

On voit bien, à travers cet exemple, comment s'opère le déplacement entre le particulier et le général, qui permet la condamnation morale de la Compagnie tout entière. En mettant en scène la figure du fondateur de l'Ordre, le texte cherche à rapporter les pratiques déviantes mais singulières de quelques auteurs jésuites, à la force d'une tradition ${ }^{72}$, aux logiques profondes de l'institution. L'usurpation, le plagiat, le vol de manuscrit ne constituent pas seulement des entraves à la propriété littéraire naissante, mais sont l'adaptation dans l'univers littéraire, des nombreux topiques du complot. Ainsi, dans le mouvement de va-et-vient entre la singularité des exemples et la personne collective de l'Ordre se dessine une identification de l'activité littéraire au répertoire des actions condamnées de la Compagnie de Jésus.

Loin d'être simplement anecdotique, cette production satirique ou pamphlétaire a eu des effets négatifs et contre-publicitaires, qui ont pu inciter les supérieurs à circonscrire le travail d'écriture dans des limites précises à l'intérieur de l'Institution et à le maintenir en situation de dépendance à l'égard des autres apostolats mieux établis. Cette prolifération de « mauvais discours », dont il faudrait plus finement analyser la chronologie, continue d'alimenter un imaginaire du complot. La littérarisation des polémiques antijésuites, par le biais d'une fabrication de stéréotypes littéraires, d'un usage répandu de la fiction et de l'utilisation de formes éditoriales attractives, favorise ainsi une circulation plus intense de ces livres et de ces thèmes dans le royaume.

Stéphane Van Damme (janvier 1999).

71. Ibid., p. 13.

72. La figure d'Ignace de Loyola auteur fait l'objet d'une double interrogation. D'une part, on souligne les limites d'une assignation simple et univoque des textes fondateurs de l'Ordre (Constitutions, Récit, etc.) à la paternité littéraire d'Ignace; d'autre part, une hypothèque pèse encore sur l'originalité de la spiritualité jésuite que l'on tend à rapporter à divers emprunts, en particulier aux courants spirituels réformateurs du début du $\mathrm{Xvr}^{e}$ siècle. Sur ces débats historiographiques, on renvoie aux présentations de ces différents textes in IGNACE DE Loyola, Écrits, op. cit. supra n. 52. 